\title{
Operationally complete work done suggests presence of extra potentials corresponding to repulsive forces
}

\author{
Jakub Czajko \\ Science/Mathematics Education Department, Southern University and A \& M College, \\ Baton Rouge, LA 70896, USA \\ E-mail address: sunswing77@netscape.net
}

\begin{abstract}
Operationally complete representation of work done and the corresponding to it potential energy within the usual radial/center-bound, nonrotating gravitational force fields comprises two extra terms: linear nonradial and angular nonradial, in addition to the usual radial term. Since these nonradial terms have negative signs, they suggest presence of potentials corresponding to repulsive forces generated by the very same, usual radial attractive force field. The extra linear nonradial term depends on exposure of an orbiting satellite to the distribution of mass within the field, whereas the extra angular nonradial term also depends on that as well as on exposure of the satellite to density of matter of the mass source that generates the usual, locally dominant radial/center-bound attractive gravitational force field.
\end{abstract}

Keywords: Nonradial potential energy; work done; repulsive potential of gravity

\section{INTRODUCTION}

Stability of macroscopic solids can indicate [the necessity of] presence of some repulsive gravitational forces [1]. If so then perhaps we missed something (inherent to force fields) which warrants the fleeting stability that is visible in nature, especially on macro scale. I will show below that definitely repulsive potentials emerge in the usual radial/center-bound gravitational force field as a necessary mathematical consequence of the field ${ }^{\text {ee }}$ s abstract geometric structure that is imparted by interacting potentials of both masses. Since abstract potentials correspond to potential energy spent on the work done by the given force field on a satellite orbiting the field (or on a body traversing it), the geometric structure is of both physical and mathematical provenience tied together. Lobachevski already emphasized that [2].

In 1898 AD Schuster has conjectured that chemical elements with negative masses should be repelled by [the usual radial central] gravitational force field [3]. Nevertheless, I 
shall show in what follows that interacting radial potentials can also produce additional nonradial potentials with signs opposite to the usual attractive radial potential, which would correspond thus to a repulsive force. Yet the possibility of existence of nonradial potentials was ignored in former physics.

Even when decomposed into the three-dimensional (3D) coordinate system $(X, Y, Z)$, the [generic] potential function $\mathrm{V}(\mathrm{r})$ of an attractive radial/center-bound force field due to mass sources was considered only in the radial direction $[4,5]$.

And even when it was generalized to $\mathrm{n} \geq 3$ dimensions, the potential was still defined via $n$ iterated integrals of exactly the same, single generic radial potential function [6]. No other than radial potentials were recognized in Newtonian and Einsteinian theories of gravitation. As a matter of fact, Einstein explicitly declined to consider tangential contributions to the radial impact of gravitational field [7].

Contrary to common perception, center-bound force field is not static during interactions with traversing it bodies. It does not remain the same once it has been created even when its source does not alter any of its field-related attributes. Even if the field ${ }^{\text {ee }}$ source remains unchanged, the field ${ }^{\text {ees }}$ interaction/behavior depends also on all the other entities that happen to move through the field, even if their possible contribution to the field is negligibly small. Although as a whole it could theoretically last forever, the radial field is ever rearranging its abstract internal structure. For it is not really the resultant force vector that actually does act on the particle that moves in it, but each of its coordinate subcomponents (if cast in the reference frame of the given particle affected by the central force field) acts quite independently of all its other coordinate subcomponents - compare [8].

Neglecting the inherent dynamics of the radial force field did lead to several misconceptions in the past, one of which was the former denial of existence of nonradial effects of the usual radial force fields [9]. However, actual existence of nonradial effects and thus associated with them nonradial potentials is confirmed in few experiments [10] and is mathematically necessary [11]. Nevertheless, fairly substantial heap of misconceptions, which emerged in the former physics, has its roots in misguided applications of mathematics, regardless of whether it was done by physicists or mathematicians. Thence a (new) synthetic mathematics is being developed. The new mathematics can invent or synthesize new laws by enhancing those already existing ones by taking cues from curious experimental hints [12].

\section{NEW SYNTHETIC MATHEMATICS FOR PHYSICAL SCIENCES}

Physics could come up with some new laws of nature and even ensure that they conform to experimental data, but to ascertain that the new laws truly reflect defining features of the reality they describe requires also conceptually sound, as well as operationally complete, (new) synthetic mathematics, which is envisioned not only as a tool to confirm existing physics, but as guide toward new ideas too.

I have derived (from experimental hints) new law governing nonradial effects of radial fields [10]. The law retrodicted few previously unexplained experimental results [10]. But that law was neither sufficiently detailed nor general enough to retrodict all related effects nor did it include also some other variables that could influence the phenomena ruled by the law yet were absent in that experiment-driven formula. This has eventually been accomplished in [12] after removing few mathematical obstacles that kept the law from being revealed earlier [9], [13]. In the sense the new synthetic mathematics is necessary to enhance laws of physics. 
Although quantum mechanics significantly advanced operational methods of mathematics, it has also inherited several old conceptual deficiencies of the latter, stemming from the subservient role (often termed as that of "handmaid") ascribed to mathematics by physical sciences. But mathematics has significant, though yet untapped, predictive capabilities whose power extends far beyond the imagination of nonmathematicians and of some mathematicians as well. Through the abstract "eye" of mathematics one might fathom unknown yet, higher laws of nature and mathematics and then actually devise them based upon set of proven operational principles of mathematics [12]. Laws of physics/nature belong in mathematics, not physics, which merely interprets them. Interpretation is important, but it may not be able to reveal the extent to which the scope of the law could be stretched.

But in order to stay relevant to physical applications and to the reality we live in, mathematics should take into account also the unanticipated experimental hints that suggest existence of previously unforeseen features of the reality.

Instead of leading the efforts to attain predictive enhancements of the already known laws in all sciences (itself included), mathematics concentrated on proving that its deductions are consistent with its own (yet not always forward-looking) assumptions about the realities they pertain to. With its focus directed inwards, mathematics virtually lost its natural leadership role among exact sciences, which it enjoyed since Newton"s invention (and Leibniz"s "redemption") of differential calculus. Rather than upgrading its axiomatic bases upon those experimental hints that indicate ever-expanding features of physical reality, mathematics succumbed to the invidious task of restraining enhancements to the reality by tacitly defining away conceptually inconvenient or just difficult to grasp features of the reality.

Mathematics has reduced its role to just confirming what physical sciences happen to discover, even if the discovery was only a tip of the proverbial iceberg but the still submersed (hence not yet fully visible/recognized) iceberg turned out to be very different than its tip, at the expense of its integrity. Mathematics often compromised its methods and even defied some of its own proven operational laws, which tendency led to severe scientific misconceptions in the past [9].

Case in point: In 1773 AD Lagrange realized that Newtonian gravitational force $\mathbf{F}$ equals to gradient $\nabla \mathrm{V}=-\mathbf{F}$ of the generic scalar potential function $\mathrm{V}=1 / \mathrm{r}$ that solves Laplace equation [14]. It was like discovery of a raw mathematical "diamond" that was left unpolished. Mathematics not only invented fake theorem that kept the idea from being further investigated, but it even tacitly violated its own proven laws/rules [9] and suppressed its own proven achievements [15] when they disagreed with some man-made mathematical ideas about the reality.

The generic potential is evidently identical to normal/radial curvature of free space, however. Realizing this, one might ask: If so then how about torsion that is actually the second curvature (orthogonal to the primary one), which is (according to differential geometry) absolutely necessary to be considered in general [13]? A single solution of a differential equation is not necessarily its most comprehensive solution. Traditional approach to physical problems used mathematics selectively. It just ignored issues considered as conflicting with formerly accepted paradigms.

Despite having knowledge (from introductory level calculus courses) that if the generic rate $\mathrm{dW}$ of work done $\mathrm{W}$ shall be represented by inner product of the vectors of force $\mathbf{F}$ and distance $\mathbf{r}$, then according to proven [16] and operationally complete (hence mandatory) product differentiation rule/law the rate ought to be rendered as $\mathrm{dW}(\mathbf{F} \bullet \mathbf{r})=\mathbf{F} \bullet \mathrm{d} \mathbf{r}+\mathbf{r} \bullet \mathrm{d} \mathbf{F}$ rather than simply as $\mathrm{dW}:=\mathbf{F} \bullet d \mathbf{r}$ as it used to be defined in both former physics and 
mathematics. Although the clearly incomplete formula is admissible just for a single standalone force whose magnitude does not vary when the magnitude of distance $r=|\mathbf{r}|$ is fixed, for radial/center-bound force fields the cut-short formula is inadequate and leads to incorrect predictions [9].

With more than one source of a force field, the effective vector of force can vary even when the distance is fixed. Hence if distinct sources of the field could not be meaningfully amalgamated into as if single-source radial local field, the incomplete (and thus law-defying) traditional formula is wrong. Notice that the right-hand side (RHS) term rødF has evidently nonradial character because the distance $r$ is fixed there. The fixed radial distance $r$ is just a parameter (in the RHS term) determining generic equipotential surface that surrounds the local gravity center of the locally dominant radial/center-bound force field, i.e. the (central) field generated by the main, single (or amalgamated) local mass source alone. I will show in what follows that the neglected nonradial term can change structure of the entire potential function and thereby also our understanding of gravity.

One could imagine several directly or indirectly independent variables upon which the varying rate of force function $\mathrm{d} \mathbf{F}()$ has to depend, one of which should vary along the equipotential surface, in order for the mathematics (to be deployed for handling the varying force) to make also conceptual sense. Before tackling the issue, however, we must clarify the necessary contextual framework.

\section{NONRADIAL EFFECTS OF RADIAL/CENTRAL FORCE FIELDS}

It has been shown that presence of some other than radial (hence nonradial) components of the usual radial/center-bound force fields really is mathematically unavoidable [13]. Therefore from operationally correct definition of work done rate containing nonradial terms, this fairly comprehensive formula emerged [9]:

$$
\mathrm{dW}=\mathrm{d}(\mathbf{F} \bullet \mathbf{r})=\mathbf{F} \bullet \mathrm{d} \mathbf{r}+\mathbf{r} \bullet \mathrm{d} \mathbf{F}=-\mathrm{F} \cos 2 \alpha \mathrm{dr}+2 \mathrm{Fr} \sin 2 \alpha \mathrm{d} \alpha-\mathrm{r} \cos 2 \alpha \mathrm{dF}
$$

where the equipotential term in the middle on the RHS of eq. (1) depends on the varying planar spherical angle $\alpha$ of visibility of the path pointed to by the pointing vector $\mathbf{r}$ whose value $r=|\mathbf{r}|$ is also the distance between the masses $M$ and $\mathrm{m}$.

Here $\cos \alpha=r_{p} / r$ with $r_{p}$ as the radius of perihelion and $F=|\mathbf{F}|$ the magnitude of force. Since the path was assumed as being originally straightlinear before it was twisted by the local force field, its angle of visibility $\alpha$ can be presumed as planar spherical. At the present depth of inquiry, only motion-independent (hence as if static) nonradial effects are taken into account. They happen along equipotential surfaces where the usual radial potential always remains constant by definition.

The angular (middle) term with d $\alpha$ that is determined by the angle $\alpha$ in the eq. (1) yields the equipotential function of the work done rate $\mathrm{w}_{\mathrm{rQ}}(\mathrm{Q}(\lambda))$ for which the given field $\mathrm{s}$ potential energy is spent solely along the equipotential surfaces determined by radius $\mathbf{r}$ and codetermined by the field's density of matter Q [12]:

$$
\mathrm{W}_{\mathrm{rQ}}(\mathrm{Q}(\lambda))=\frac{-4 \mathrm{kGMmr} \mathrm{p}_{\mathrm{p}}^{2} \lambda}{3 \mathrm{Qr}^{4}}
$$


which depends on the function $\mathrm{Q}(\lambda)$ measuring exposure of the orbiting or passing by mass $\mathrm{m}$ (assumed as being insignificantly small/negligible in comparison to the, assumed as huge, mass $\mathrm{M}$ ), which traverses the locally dominant gravitational force field, to the average/constant matter density $\mathrm{Q}$ of the source mass $\mathrm{M}$.

In other words: the (generally double-curvilinear) angular distance variable $\lambda=\mathrm{r} \theta$ (representing a certain spherical angle $\theta$ ) is measured along the equipotential surface that is codetermined by the average density of matter Q, i.e. the specific gravity of the source mass $M$ that generates the locally dominant gravitational field. The exposure $Q(\lambda)$ (of the mass $\mathrm{m}$ traversing the field due to the mass $M$ ) to the field ${ }^{\text {ee }}$ density of matter $\mathrm{Q}$ depends on the equipotential angular distance $\lambda$, but - at the present depth of inquiry - not on a possible evolution in space/time of the matter density function $\mathrm{Q}()$ itself.

Since in general the equipotential angular distance $\lambda$ can be curvilinear, the corresponding to it angle $\theta$ is thus spheroidal spherical one. The negative function $\mathrm{w}_{\mathrm{rQ}}(\mathrm{Q}(\lambda))$ causes energy/frequency decrease [10]. The coefficient $\mathrm{k}$ (to be found from experiments) is needed, indeed, to cover possible impact of a few expected, but unknown yet and thus unaccounted for variables; this shall be explained in more detail elsewhere. For the eq. (2) presumed nonrotating fields and masses, and assumed constant $\mathrm{G}, \mathrm{M}, \mathrm{m}, \mathrm{Q}$, and $\mathrm{r}_{\mathrm{p}}$, some of which may, nonetheless, vary depending on certain independently varying variables which are not investigated yet at present depth of inquiry into the gravitational phenomena. For the physical reality we live in is not restricted by what our imagination is able to fathom.

The eq. (2) can be rewritten in terms having more direct physical relevance

$$
\mathrm{w}_{\mathrm{rQ}}(\mathrm{Q}(\lambda))=\frac{-\mathrm{kuGMm} \lambda}{\pi \mathrm{r}_{\mathrm{p}} \mathrm{Qr} \mathrm{r}^{4}}=\frac{-\mathrm{kGMm} \lambda}{\pi \mathrm{r}_{\mathrm{p}} U \mathrm{r}^{4}}=\mathrm{w}_{\mathrm{Q}}\left(\mathrm{r}, \mathrm{r}_{\mathrm{p}}, \circlearrowright, \lambda\right)
$$

where $v$ is perimeter volume enclosed by the distance $r_{p}$ to the perihelion from the gravity center of the locally dominant force field [17]. These two equations (2) and (3) are conceptually equivalent, but the eq. (3) is cast in the larger context of purely geometric terms (volume and circumference of an idealized approximately circular orbit, or more likely a part of the orbit as it is seen inside the perihelion).

Specific gravity $\mathrm{Q}=\mathrm{q}_{\mathrm{M}} / \mathrm{q}_{\text {water }}$ is an intensive (hence comparative) measure of density of matter of the source mass $M$ relative to density of water. Therefore the $\delta=Q / v$ denotes relative density of matter enclosed within the perimeter volume. The sack $\mho$ can be used for comparing interactions when either the mass density of the source mass $M$ changes (as it can do in exploding or collapsing stars, for instance) or if the perimeter delineated by (shifting/moving) perihelion is altered.

The work done rate function $\mathrm{w}_{\mathrm{rQ}}(\mathrm{Q}(\lambda)$ ) that has been reparametrized into one depending on few more variables: $\mathrm{w}_{\mathrm{Q}}\left(\mathrm{r}, \mathrm{r}_{\mathrm{p}}, \widetilde{\Omega}, \lambda\right)$ could also become more detailed function $\mathrm{w}_{\mathrm{Q}}\left(\mathrm{r}, \mathrm{r}_{\mathrm{p}}, \widetilde{\sigma}, \lambda, \mathrm{M}, \mathrm{m}\right)$ if these masses would vary too. The subscript $\mathrm{Q}$ in $\mathrm{w}_{\mathrm{Q}}$ means parameter codetermining the function $\mathrm{w}_{\mathrm{Q}}()$ even if it is implicit or absent there. Symbols in parentheses following function names identify independent variables. Hence in $\mathrm{w}_{\mathrm{rQ}}(\mathrm{Q}(\lambda)) \mathrm{r}$ and $\mathrm{Q}$ play only the role of parameters, just as in $\mathrm{w}_{\mathrm{rQ}}$, but when $\mathrm{Q}$ is considered as a function $\mathrm{Q}()$ then in the latter incarnation - it is also representing the proxy variable $\mathrm{Q}(\lambda)$ that depends on the angular distance $\lambda$ and signifies exposure (along the angular distance) to the (assumed as average and thus constant) density of matter Q. Note that - at a deeper than this present level of inquiry - the function Q() can exhibit dependence also on other variables.

Parameters can be listed among independently varying variables even if they are fixed for the particular context as constants or averages determining the given situation at hand, in 
which case one might assume that they could vary in general (i.e. in certain other contexts/situations). When the variables are tentatively used as fixed parameters, however, they must not be differentiated or integrated within the same conceptual context defining the situation from which they emerged. This demands distinguishing active variables from passive parameters by unambiguous qualifiers, which was not always accentuated in former physics and mathematics. The distinction is conceptually important to avoid performing illegal operations.

\section{FORMER FIELD-NOTIONS SHOULD BE QUALIFIED AS RADIAL}

Let us review and then rectify several inconsistencies in former physics. The scalar magnitude $\Gamma=|\boldsymbol{\Gamma}|$ of the radial Newtonian gravitational force field vector $\Gamma$ as function of the (radial) distance $\mathrm{r}=|\mathbf{r}|$ between gravity centers of two interacting masses $\mathrm{M}$ and $\mathrm{m}$ was traditionally defined in physics as

$$
\Gamma(\mathrm{r}):=|\Gamma(\mathbf{r})|=-\frac{\mathrm{GMm}}{\mathrm{r}^{2}} \Rightarrow \frac{\mathrm{d} \Gamma(\mathrm{r})}{\mathrm{dr}}=\frac{2 \mathrm{GMm}}{\mathrm{r}^{3}}
$$

in the usual, attractive radial/center-bound gravitational force field. Evidently the presumed as locally dominant mass $M$ curves the local space towards itself here and that is why relativistically speaking - the smaller mass $\mathrm{m}$ is forced to orbit the locally dominant mass $\mathrm{M}$. The mass $\mathrm{M}$ can also appear as if just bending the trajectory of the mass $\mathrm{m}$ if the orbital radius tends to infinity, of course.

The radial-only (i.e. partial) rate $\partial \mathrm{W}(\mathrm{r})$ of the work done by the field on the traversing it test mass $m$ (which could be moving - in general - under influence of both: the locally dominant gravitational force field and some other, distinct fields) can be defined in terms of either the radial force vector $\Gamma(r)$ or the radial potential energy function $U(r)$, or the (physical) radial potential function $\mathrm{V}(\mathrm{r})$ as:

$$
\partial \mathrm{W}(\mathrm{r}):=\boldsymbol{\Gamma}(\mathbf{r}) \bullet \partial \mathbf{r}=\nabla \mathrm{U}(\mathrm{r}) \bullet \partial \mathbf{r}=\frac{\partial \mathrm{U}(\mathrm{r})}{\partial \mathrm{r}} \mathrm{dr} \equiv \mathrm{dU}(\mathrm{r}) \Rightarrow \mathrm{U}(\mathrm{r})=\frac{\mathrm{GMm}}{\mathrm{r}}
$$

where the physical radial potential $\mathrm{V}(\mathrm{r})$ function of the field due to mass $\mathrm{M}$ is

$$
\mathrm{V}(\mathrm{r})=\frac{\mathrm{M}}{\mathrm{r}}=\mathrm{M \kappa}=\frac{1}{\mathrm{Gm}} \mathrm{U}(\mathrm{r}) \text { where } \mathrm{U}(\mathrm{r})=\mathrm{GmV}(\mathrm{r})=\mathrm{GMm \kappa}
$$

where $\kappa=1 / r$ is generic curvature. The radial potential energy $U(r)$ of the force field relates to the radial physical potential $\mathrm{V}(\mathrm{r}$ ) (as opposed to generic potential which is identical to curvature of the orbit or path around the mass $\mathrm{M}$ ) in a very similar manner as linear momentum vector $\mathbf{p}=\mathrm{mv}$ is related to the velocity vector $\mathbf{v}$ of the orbiting (or just passing by) test mass $\mathrm{m}$.

All these former notions $\mathrm{W}(\mathrm{r}), \Gamma(\mathrm{r}), \mathrm{U}(\mathrm{r}), \mathrm{V}(\mathrm{r})$ make perfect sense, but only when they are qualified as radial. These field-based notions could be confusing, however, if left unqualified. Examples of such a confusion will be shown below.

The qualifier ,radiale suggests, however, that perhaps some other than radial (i.e. nonradial) field-based notions may also exist beside the radial ones. The sad fact that former physics ignored them did not make the nonradial effects extinct. 
Yet another indicator of necessary presence of certain definitely nonradial notions would be inconsistency of purely radial notions if used alone in general situations of any motions within the radial/center-bound force fields. Although rarely mentioned, this is indeed the case for arbitrary motions within such fields.

If magnitude $F=|\mathbf{F}|$ of an arbitrary (hence also standalone) force $\mathbf{F}$ defined in dynamical terms can be written as generic function of an arbitrary motion (which is not necessarily driven by the locally dominant radial/center-bound gravitational force field alone, but may be influenced also by some other fields) was given by

$$
\mathrm{F}(\mathrm{r}, \mathrm{t}):=\mathrm{ma}=\mathrm{m} \frac{\mathrm{d}^{2} \mathrm{r}}{\mathrm{dt}^{2}}=\mathrm{m} \frac{\mathrm{d}}{\mathrm{dt}}\left(\frac{\mathrm{dr}}{\mathrm{dt}}\right)=\mathrm{m} \frac{\mathrm{dr}}{\mathrm{dt}} \frac{\mathrm{d}}{\mathrm{dr}}\left(\frac{\mathrm{dr}}{\mathrm{dt}}\right)=\mathrm{mv} \frac{\mathrm{dv}}{\mathrm{dr}}=\mathrm{p} \frac{\mathrm{dv}}{\mathrm{dr}}
$$

then the arbitrary force $\mathbf{F}(\mathrm{r})$ is not really equal to the radial force of gravitation $\Gamma(\mathrm{r})$ unless $\mathbf{a}=$ $-\mathbf{a}_{\mathrm{r}}$, i.e. unless the arbitrary acceleration, , $\mathrm{a}^{\mathrm{ee}}$ is radial, which only happens when all forces can be amalgamated into as if single force field. Hence

$$
\mathrm{F}(\mathrm{r}, \mathrm{t}):=\mathrm{ma} \neq \Gamma(\mathrm{r}):=-\mathrm{m} \frac{\mathrm{GM}}{\mathrm{r}^{2}}=-\mathrm{ma}_{\mathrm{r}}
$$

which shows that former balancing of forces was not always correct. If qualified, the eq. (8) indicates need to take also other than radial forces to balance arbitrary forces. Former physics ignored this inadequacy. The disambiguation via explicit qualifiers provides further evidence for necessity of certain nonradial effects [11].

Engines of a spacecraft or laser pulses might create extra contributions to the effective local field, in which case the resulting field cannot be described in radial terms alone. And so does a distant star, even though its impact is detectable only through the rays it emitted, for it is most likely too far away from the local field.

Integrating the above differentials gives equivalence of kinetic energy $\mathrm{K}$ and potential energy $\mathrm{U}$, but only under the (usually unspoken) assumption that all the motions in the radial/center-bound force field are radial. Without accepting this assumption, equivalence of arbitrary kinetic and radial potential energy is invalid, and thus the alleged consistency of the so-defined energies can fall apart because motions driven by two distinct sources need not always be $\mathrm{radial} /$ center-bound:

$$
\mathrm{K}(\mathbf{x}, \mathrm{t}):=\int \mathrm{mvdv}=\mathrm{m} \frac{\mathbf{v}_{\mathbf{x}}^{2}}{2} \neq \mathrm{U}(\mathbf{r}):=-\int \frac{\mathrm{GMm}}{\mathrm{r}^{2}} \mathrm{dr}=\mathrm{m} \frac{\mathrm{GM}}{\mathrm{r}}=\mathrm{m} \frac{\mathbf{v}_{\mathrm{r}}^{2}}{2}=\mathrm{K}(\mathbf{r}, \mathrm{t})
$$

where the radial potential energy $U(r)$ equals only to radial kinetic energy $\mathrm{K}(\mathbf{r})$ but these two are not equal to arbitrary kinetic energy $\mathrm{K}(\mathbf{x})$ which needs not be radial in general (over a distance $\mathbf{x}$ determined by a pointing vector $\mathbf{x}$, which is not always radial). It is known that magnitude/intensity of a field at a point does not determine completely the field at that point [18]. Hence kinetic energy of a mass $m$ driven by more than one source of the effective field may not always be radial: $\mathrm{K}(\mathbf{x}) \neq \mathrm{K}(\mathbf{r})=\mathrm{U}(\mathrm{r})$. Mercier already realized that there must be some mixed terms of potential energy [19], [20], even though he has not identified them exactly.

If despite the inequality of arbitrary kinetic energy $\mathrm{K}(\mathrm{x})$ and radial potential energy $\mathrm{U}(\mathrm{r})$ energy should be conserved, an extra energy must exist in order to make/fill up the gap. Chow has pointed out that velocity-dependent interaction is noncentral and thus clearly nonradial [21]. So the possibility of existence of other than radial effects was always present, but it was 
routinely ignored [22]. Although one can always drop some negligibly small nonradial terms in an approximation, if a formula is supposed to be conceptually correct then it must be complete.

Even when velocity of the test body that crosses the local force field always remain constant, certain clearly nonradial effects can occur anyway. This is fairly well visible when a nominally massless particle like photon travels at the speed of light and tangentially passes near star such as our Sun, or when some radio waves passed along practically equipotential surface of the Earth [10].

Although the changing distance (to our Sun) from the rays (coming to Earth from distant stars) that pass near our Sun is radial, their trajectory path $\mathrm{x}$ along which energy is exchanged (between the rays and the Sun 'es force field) via work done by the Sun se field is not exclusively radial. Their path can have both radial components and also components that are not radial with respect to the Sun [10].

The incompletely defined work done could be consistent only if all the force field vectors would always be parallel (which in center-bound fields is impossible by definition), or if the force field vectors could not be resolved into parts parallel and perpendicular to the path of the moving object, but it is always possible by Hamilton "es theorem [23]. Radial-only notions cannot thus guarantee consistency.

I have showed that a certain nonradial work done (and the corresponding to it nonradial potential energy) as well as an extra nonradial force produced by the work/energy emerges from other than radial interactions within the usual central radial/center-bound force field.

We have also seen that former radial-only notions cannot account for all field interactions. Also some unanticipated experimental results strongly indicated the presence of definitely nonradial effects of the usual radial gravitational force field, which depend not only on the local fielde $s$ (mass source ${ }^{\text {ee }}$ ) density of mass, but inversely also on (an exposure of the orbiting satellite/body to) the local field"s density of matter [9,10,17].

\section{COMPLETE WORK DONE AND TOTAL POTENTIAL ENERGY}

At the present depth of inquiry into gravitational phenomena happening in a locally dominant radial/center-bound gravitational force field I am assuming (for the sake of simplicity of this presentation) that all masses of interacting bodies remain constant during their gravitational interaction.

This latter assumption means that the gravitational force depends primarily only on the radial distance from the gravity center of the given local force field.

Henceforth the term ,lineare, when used in the phrase „,nonradial linear"e, can mean „straightlineare (as opposed to ,equipotential angulare, which is bound to an equipotential surface) as well as „aurvilinear"e, if the latter is not surface-bound.

$$
\begin{gathered}
\text { Since } \cos 2 \alpha=\left(2 \cos ^{2} \alpha-1\right)=\left(2 \frac{\mathrm{r}_{\mathrm{p}}^{2}}{\mathrm{r}^{2}}-1\right) \text { we get from the eqs. (1) and } \\
\mathrm{dW}(\mathbf{F} \bullet \mathrm{d} \mathbf{r}):=-\mathrm{F} \cos 2 \alpha \mathrm{dr}=\frac{\mathrm{GMm}}{\mathrm{r}^{2}}\left(2 \frac{\mathrm{r}_{\mathrm{p}}^{2}}{\mathrm{r}^{2}}-1\right) \mathrm{dr}=\frac{2 \mathrm{GMmr}}{\mathrm{r}^{4}} \mathrm{dr}-\frac{\mathrm{GMm}}{\mathrm{r}^{2}} \mathrm{dr}
\end{gathered}
$$

which is the usual, radial linear part of total work done rate [9]. After integration we obtain two respective amounts of the (radial linear) part of the total work done 


$$
\mathrm{W}(\mathbf{F} \cdot \mathrm{d} \mathbf{r})=\int_{\mathrm{r}}^{\infty} \mathbf{F} \cdot \mathrm{d} \mathbf{r}=\int_{\mathrm{r}}^{\infty}\left(\frac{2 \mathrm{GMmr}}{\mathrm{r}^{4}}-\frac{\mathrm{GMm}}{\mathrm{r}^{2}}\right) \mathrm{dr}=\frac{2 \mathrm{GMmr}}{3 \mathrm{r}^{3}}-\frac{\mathrm{GMm}}{\mathrm{r}}
$$

where the radial potential that corresponds to the radial part of the work done rises from $r$ to infinity [12]. Notice that the perihelion radius is always smaller than the varying radius/distance $\left(r_{p} \leq r\right)$ by definition and is assumed here as being constant.

Similarly also the nonradial linear work done rate is given by the expression:

$$
\mathrm{dW}(\mathbf{r} \cdot \mathrm{d} \mathbf{F})=-\mathrm{rcos} 2 \alpha \mathrm{dF}=-\mathrm{r}\left(2 \frac{\mathrm{r}_{\mathrm{p}}^{2}}{\mathrm{r}^{2}}-1\right) \frac{2 \mathrm{GMmdr}}{\mathrm{r}^{3}}=\left(\frac{2 \mathrm{GMm}}{\mathrm{r}^{2}}-\frac{4 \mathrm{GMmr}}{\mathrm{r}^{4}}\right) \mathrm{dr}
$$

and its integration gives us two amounts of the nonradial linear part of work done

$$
\mathrm{W}(\mathbf{r} \cdot \mathrm{d} \mathbf{F})=-\int_{0}^{\mathrm{F}} \mathrm{r} \cos 2 \alpha \mathrm{dF}=\int_{\mathrm{r}}^{\infty}\left(\frac{2 \mathrm{GMm}}{\mathrm{r}^{2}}-\frac{4 \mathrm{GMmr}_{\mathrm{p}}^{2}}{\mathrm{r}^{4}}\right) \mathrm{dr}=\frac{2 \mathrm{GMm}}{\mathrm{r}}-\frac{4 \mathrm{GMmr}}{3 \mathrm{r}^{3}}
$$

which complements the radial work done. Notice that while the radius $r$ grows to infinity the magnitude $\mathrm{F}$ of the force diminishes, but the minus sign reverses it.

It has also been shown that amount $\mathrm{W}_{\mathrm{rQ}(\lambda)}$ of nonradial angular work done is

$$
\mathrm{w}_{\mathrm{rQ}}(\lambda)=\frac{-4 \mathrm{kGMmr}}{\mathrm{p} \lambda}=\frac{-\mathrm{kuGMm} \lambda}{3 \mathrm{Qr}^{4}}=\frac{-\mathrm{kGMm} \lambda}{\pi r_{\mathrm{p}} \mathrm{Qr} \mathrm{r}^{4}}
$$

where $v$ is the perimeter volume enclosed by the distance $r_{p}$ to perihelion from the local gravity center of the mass $M[12]$, with the angular distance $\lambda=r \theta$ runs along equipotential surface codetermined by the radial distance $r$ and spherical angle $\theta$.

Since specific gravity $\mathrm{Q}=\mathrm{Q}_{\mathrm{M}} / \mathrm{Q}_{\text {water }}$ is conventionally defined as an intensive (i.e. comparative) measure of density of matter of the source mass $M$ relative to density of water under normal conditions, the parameter $\mho=\mathrm{Q} / \mathrm{v}$ yields the unit of relative density of matter enclosed inside the perimeter volume $v=4 \pi\left(\mathrm{r}_{\mathrm{p}}\right)^{3} / 3$ [17].

The coefficient $\mathrm{k}=1$ for perihelion close to surface of the source mass $M[12,17]$. I have successfully reconciled experiments with waves passing near Sun and Earth using the value $\mathrm{k}=1$ [10], but $\mathrm{k}$ should be determined from experiments for, in general, it could depend on perihelion and perhaps on a few other factors too. As in eq. (1) we got three distinct components: the usual radial linear term $\mathrm{W}(\mathbf{F} \bullet \mathrm{d} \mathbf{r})$ in $(11)$, the nonradial linear term $\mathrm{W}(\mathbf{r} \bullet$ $\mathrm{dF}$ ) in (13) and the nonradial angular term $\mathrm{w}_{\mathrm{rQ}(\lambda)}$ in (14). Taken together they give us the total amount of work done (within nonrotating radial/center-bound force fields), which - due to energy conservation law - must be equal to the total amount of potential energy $U$ that compensates the work done by the local force field on the gravitational interaction

$$
\mathrm{U} \equiv \mathrm{W}(\mathrm{d}(\mathbf{r} \bullet \mathbf{F}(\mathbf{r})))=\mathrm{W}(\mathbf{F} \bullet \mathrm{d} \mathbf{r})+\mathrm{W}(\mathbf{r} \bullet \mathrm{d} \mathbf{F})+\mathrm{w}_{\mathrm{rQ}}(\mathrm{Q}(\lambda))
$$

which can be written in terms of perimeter volume $v$ and the circumference $ц$ of the perihelion circle and a relative density of matter $\mho$ enclosed by the perihelion

$$
\mathrm{U}(\mathrm{r}, \lambda)=\frac{\mathrm{GMm}}{\mathrm{r}}-\frac{2 \mathrm{GMmr} \mathrm{r}_{\mathrm{p}}^{2}}{3 \mathrm{r}^{3}}-\frac{4 \mathrm{kGMmr} \mathrm{p}_{\mathrm{p}}^{2} \lambda}{3 \mathrm{Q} \mathrm{r}^{4}}=\frac{\mathrm{GMm}}{\mathrm{r}}-\frac{\mathrm{GMmu}}{\mathrm{r}^{3}}-\frac{2 \mathrm{kGMm} \lambda}{ц U \mathrm{r}^{4}}
$$


where the $2^{\text {nd }}$ nonradial linear term depends on the perihelion radius $r_{p}$ of the mass $m$ orbiting the source mass $\mathrm{M}$ of the field, as does the $3^{\text {rd }}$ purely angular nonradial term, which also depends on exposure of the mass $m$ to density of matter $Q$ of the mass source $M$ of the locally dominant field via the angular/equipotential distance $\lambda$ that is measured along the equipotential parts of a trajectory of the mass $\mathrm{m}$. The perihelion circle is not meant to suggest circular trajectory. It only alludes to the amount of matter enclosed within the volume determined by the perihelion radius.

Since the newly emerged measure, namely volume per circumference $\mathrm{y}=/ ц$ is purely structural geometric quantity, which seems to play very important role in linear nonradial potentials, I shall call it henceforth volpercirc, whereas its inverse (circpervol $1 / \mathrm{y}=ц / \mathrm{v}$ ) clearly plays defining role for angular nonradial potentials.

The total amount of work done and thus consequently also the corresponding to it potential energy of the locally dominant radial/center-bound gravitational force field indicates presence of two relatively small gravitational repulsion terms generated by the usual attractive radial field, due to these two negative nonradial components (linear and angular, respectively) whose signs are opposite to the usual radial potential that generates attractive force between the masses $\mathrm{M}$ and $\mathrm{m}$.

If the test mass $m$ would orbit the (assumed as huge) mass $M$ on a circle $r=r_{p}$

$$
\mathrm{U}(\mathrm{r}, \lambda)=\frac{\mathrm{GMm}}{\mathrm{r}}-\frac{2 \mathrm{GMm}}{3 \mathrm{r}}-\frac{4 \mathrm{kGMm} \theta}{3 \mathrm{Qr}}=\frac{\mathrm{GMm}}{\mathrm{r}}\left(\frac{1}{3}-\frac{4 \mathrm{k} \theta}{3 \mathrm{Q}}\right)
$$

then potential energy $U$ of such an idealized system would increase hyperbolically with radial distance. With increasing radial distance from gravity center, the radial potentials surpass the nonradial ones, which are significant only near surface of the mass $M$ that generates the locally dominant gravitational field - see Fig. 1.

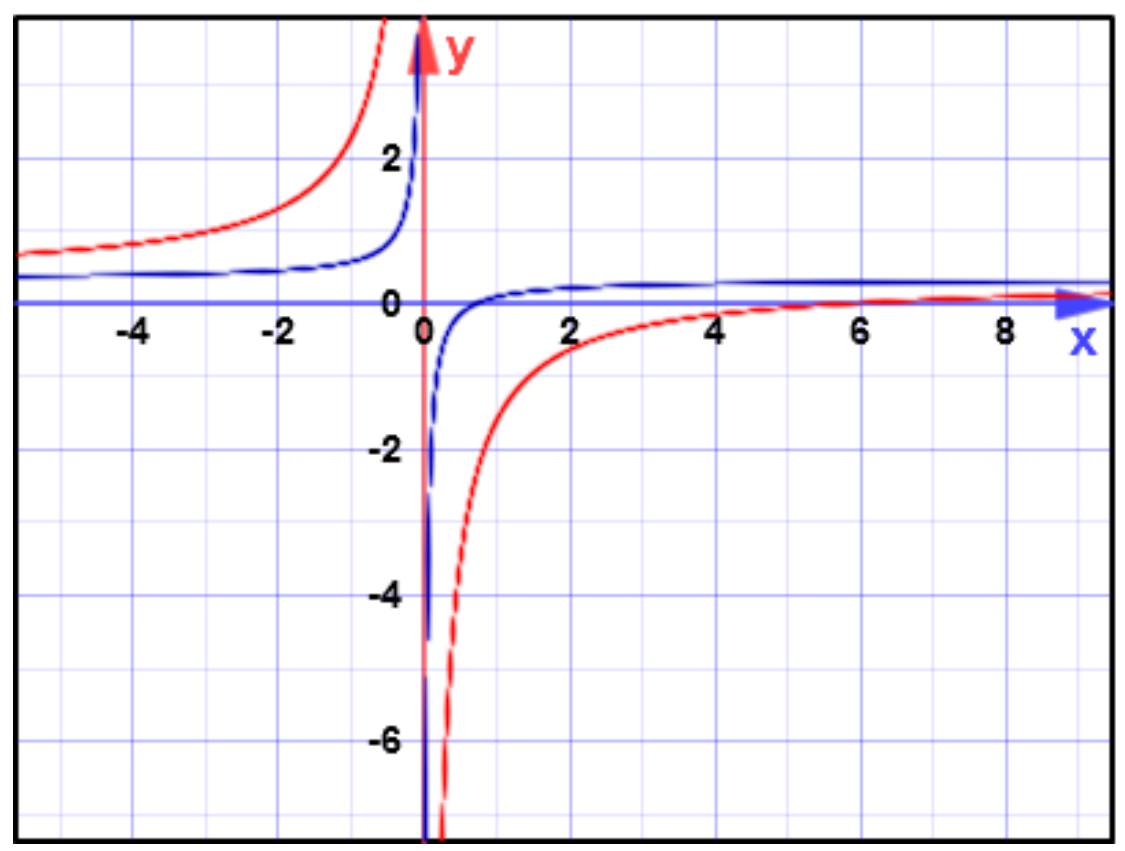

Fig. 1. Simplified plot of cross section of the hyperbolaes right-branch - eq. (17). 
Notice that we are talking here about the total potential energy involved in gravitational interactions, not about potential energy of the whole field, which is just one (even though certainly the most important one) of several factors directly relevant to interactions happening within the given gravitational field.

Yet because presence of nonradial twist (and thus some $\theta$ ) is mathematically unavoidable [11], effects of nonradial potential energy of the field ${ }^{\text {ee }}$ s interactions cannot be ignored. For mass-sources of the local field with relatively high density of matter the rapidly increasing negative hyperbolic cross-sections on the RHS of the eq. (17) evidently flatten faster with increasing radial distance - see Fig. 1.

The plot in Fig. 1 shows total potential energy (y) of interactions increasing with the radial distance $r=x$ for Earth (blue) with $Q \approx 5.52$ and Saturn (red) with $Q \approx 0.687$. This plot represents the eq. (17) approximated for these two planets.

Yet if the eq. (17) must retrodict the radial Newtonian gravity, the condition

$$
\frac{\mathrm{k} \theta}{\mathrm{Q}}=1 \Rightarrow \mathrm{k}=\frac{\mathrm{Q}}{\theta}
$$

is necessary, but only as a limit. It is up to experiments to determine the $\mathrm{k}$, which could be a function of several other factors. Since the (minimal) angle $\theta$ (or the induced nonradial twist as it might be called) could vary even when the average density of matter Q remains constant, therefore - at the present depth of inquiry -the coefficient $\mathrm{k}$ may vary too. This seemingly confirms the fact that there can be no absolutely empty physical space and no infinitely small singularity possible.

Two unbiased experiments have been reconciled with $\mathrm{k} \cong 1$ with nonradial effects happening fairly close to surfaces of the Sun and Earth [10]. If the angle $\theta$ is tied to the density of matter $\mathrm{Q}$ of the mass source of the local force field, the field should exhibit an intrinsic rotation, which is neglected for the time being. These tentative conclusions agree with realistic interpretation of infinity [24], [25]. These and other related issues shall be discussed in more detail elsewhere.

\section{NONRADIAL POTENTIALS COMPLEMENT THE RADIAL ONE}

While Newton, Einstein and most subsequent investigators of gravitational phenomena usually considered the proverbial gravitating apples as being only under the influence of single-source (locally dominant or perhaps amalgamated) gravitational field and therefore as freely falling directly towards the local gravity center, the depth of their inquiry was always restricted to radial-only effects of gravitation. For Newton any exceptions from purely radial (hence center-bound) trajectory were essentially of procedural nature, i.e. caused only by a preexisting momentum of the locally bound satellite or a body passing by.

Due to lack of the notion of potential of gravitational field at the time when Newton came up with his theory of gravitation he did not see his force as arising from clash of the field ${ }^{\text {ee }} \mathrm{s}$ potentials. His greatest achievement was representing changes to physical concepts by differentials. But since Lagrange and especially after Frenet, the unavoidable nonradial potentials should not be ignored [11].

Besides, both Newton and Einstein felt justified in their rejection of possible other than radial effects of the usual radial/center-bound gravitational fields by the Galileies misguided conclusion that constitution of matter (and in particular thus density of matter) does not affect 
gravitational phenomena, which he had drawn from his too oversimplified experiments with dropping objects from the leaning tower of Pisa. Even though the simplistic Galileiees conclusion was instrumental for both Newton and Einstein, it is nevertheless mathematically unacceptable [17] and I have shown in [10] that two ingenious modern experiments defied it [17].

We should certainly applaud Galilei for easing the way for Newton to come up with his relatively simple equation for radial gravitational interactions, but we should not keep on fooling ourselves by perpetuating his mathematically deficient reasoning, which was step forward back then but is hampering physics today.

For Einstein the exceptions were due to curvature of the spacetime - hence of a structural nature. However, two distinct objects at the same point in space could have very different trajectories, depending on their preexisting momentum, which means that each object carries its own peculiar spacetime as if on its back, which fact makes them incomparable - see [26,27]. Hence the structure of Einsteinian curved spacetime seems to be flexible, as if personalized spatial structure, neither a solid nor a geometric space. Therefore its potential energy cannot be localized.

Since the extra nonradial effects have emerged from operationally complete representation of potential energy, they indeed complement the general theory of relativity (GTR) which had been deliberately restricted by Einstein to only radial gravitational interactions [7]. Besides, GTR and GTR-like theories of gravitation published prior to year $2000 \mathrm{AD}$ (i.e. before the theory of nonradial gravitational effects was envisioned in [28] and then invented in [10]), merely decomposed the magnitude of the, essentially still radial, effect of the curved spacetime (or quasi-Newtonian radial force) into the global 3D coordinates tied to local gravity center of the primary mass source that generates the locally dominant gravitational field.

The GTR's primary equations of purely radial gravitational interactions did not take into account any physical magnitudes other than those already present in the Newtonian theory, but they changed the way the radial gravitational potentials are being distributed in spacetime. All former theories of gravitation assumed that interactions within gravitational fields were determined by density of mass alone, based on the Galileiee s erroneous conclusion that density of matter does not matter.

The eq. (17), however, also shows how potentials involved in gravitational interactions are being composed (as opposed to just decomposition of radial force or radial potential onto a three-dimensional (3D) coordinate system, which still does not turn the decomposition into a composition). The distinction between composing versus decomposing vectors is very important. Riemann has already pointed out that even when decomposed into 3D coordinate system $(\mathrm{X}, \mathrm{Y}, \mathrm{Z})$, the [generic] potential function $\mathrm{V}(\mathrm{r})$ of attractive radial/center-bound force field due to mass sources was still considered only in the radial direction alone [4,5]. If force is simplistically viewed as just a passive property of radial/center-bound force field, then it is radial. But the active force involved in interactions is being composed from potentials of the interacting bodies - which fact we learned from Aharonov-Bohm effect - [17], and such active force is not always radial [11].

Decomposition is merely redistribution or deconstruction of the usual radial-only potentials - it is just a projection of the radial potentials on the coordinate axes. Composition, however, shows where their subcomponents originated from. New subcomponents could emerge in composing potentials when more defining factors are taken into consideration. Decomposing radial-only potentials cannot add any new predictors. To insist that any derived

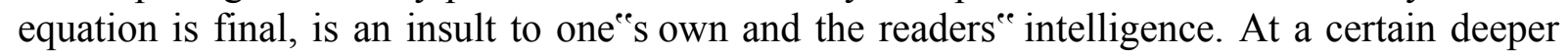


than the present level of inquiry one can find that Sun "s gravity depends also - to some degree - on its average surface temperature, for instance. More precise equation can reveal tiny dependence of gravity on the abundance of its elements, hence on the Sun's age.

Moreover, at the present depth of inquiry into gravitational interactions the eq. (17) shows the impact of exposure of the orbiting (or just passing-by) mass $m$ to (average) composition/density of matter of the field ${ }^{\text {ee }}$ s source mass $M$ (though not to the internal structural composition of particulate matter itself yet).

In the sense the nonradial theory complements radial theories of gravitation without defying their tenets. The distribution of purely radial interactions in the GTR is not questioned. All former theories of gravitation were virtually (though not always openly) challenged by the mathematical incompleteness of the faulty previous definition of potential energy and work done, which they inherited from ingenious work of Lagrange [9], likely due to his overly reliance on the aforesaid oversimplified Galileiees conclusion, I presume.

No theory can tacitly violate select (because they seem inconvenient and/or their consequences are difficult to grasp) mandatory operational laws/rules of mathematics and remain unscathed. Former incomplete mathematics resulted not only in misguided theories of physics, but it also adversely affected other related reasonings and their applications to other mathematical theories.

With deepening levels of inquiry into gravitational phenomena our previous laws of physics will grow more detailed. The old, coarse laws will be enhanced by effects revealed in new experiments, which should not be ignored in mathematics if it wants to stay relevant to reality in general and physical sciences in particular.

\section{CONCLUSIONS}

Total potential energy of gravitational field spent on work done by the radial field of gravity depends on perihelion radius/distance as well as on the curvilinear distance traveled along equipotential surfaces and also (inversely though) on the density of matter of the source mass that generates the locally dominant field.

New law for gravitational interactions in terms of work done and potential energy has been synthesized on the basis of operationally complete mathematical representation of the work done rate and upon its relation to potential energy. The new law suggests that, in addition to the usual radial gravitational potential, two extra repulsive potentials arise: one is linear nonradial and the other is angular nonradial. The extra potentials modify the former, usual radial potential function.

The operationally complete (and thus mathematically legitimate) potential energy $U(r, \lambda)$ varies not only as a result of position vector changing within the usual locally dominant radial/center-bound gravitational force field, but it is also codetermined by several other variables and fixed parameters, one of which is (angular/equipotential exposure to) average density of matter of the local field.

\section{References}

[1] Jeffreys H., Jeffreys B., Methods of mathematical physics. Cambridge: Cambridge Univ. Press, 2001, p.202. 
[2] Licis N.A., Philosophical and scientific meaning of ideas of N.I. Lobachevskii. Riga, 1976, pp. 58, 372 [in Russian].

[3] Hughes R.J., Contemp. Phys. 199334 177-91.

[4] Riemann B., Schwere, Elektricität und Magnetismus. Hannover: Carl Rümpler, 1876, p. 9ff.

[5] Gutzwiller M.C., Chaos in classical and quantum mechanics. New York: Springer, 1990, p. 101.

[6] Kasner E., De Cicco J., PNAS USA 38 (1952) 145-148.

[7] Einstein A., The Foundations of the General Theory of Relativity. [pp.111-164 in: H.A. Lorentz et al. The principle of relativity. Dover, New York 1923, see p.161].

[8] Sokolnikoff I.S., Sokolnikoff E.S., Higher mathematics for engineers and physicists. New York: McGraw-Hill, 1941, p. 218.

[9] Czajko J., Stud. Math. Sci. 7(2) (2013) 25-39.

[10] Czajko J., Chaos, Solit. Fract. 11 (2000) 2001-2016.

[11] Czajko J., Appl. Phys. Res. 3(1) (2011) 2-7.

[12] Czajko J, Stud. Math. Sci. 7(2) (2013) 40-54.

[13] Czajko J., Chaos Solit. Fract. 20 (2004) 683-700.

[14] Birkhoff G. (Ed.) A source book in classical analysis. Cambridge, MA: Harvard Univ. Press, 1973, pp.335, 360.

[15] Czajko J., International Letters of Chemistry, Physics and Astronomy 17(2) (2014) 220-235.

[16] Bers L., Calculus I. New York: Holt, Rinehart and Winston, 1967, p. $216 \mathrm{f}$.

[17] Czajko J., International Letters of Chemistry, Physics and Astronomy 11(2) (2014) 89-105.

[18] Rylov Yu.A., Sov. Phys. Dokl. 7(6) (1962) 536-538.

[19] Mercier A., Analytical and canonical formalism in physics. Amsterdam: North-Holland, 1959, p. 122.

[20] Mercier A., Speculative remarks on physics in general and relativity in particular. [pp.295-303 in: De Sabbata, V. \& Weber, J. (Eds.) Topics in theoretical and experimental gravitation physics. London: Plenum Press, 1977].

[21] Chow T.L., Classical Mechanics. New York: Wiley, 1995, p. 35.

[22] Goldstein H., Poole C., Safko J., Classical mechanics. San Francisco: Addison-Wesley, 2002, p. 4ff.

[23] Doran C., Lasenby A., Gul S., Found. Phys. 23(9) (1993) 1175-1201, see p. 1186.

[24] Czajko J., Chaos, Solit. Fract. 21 (2004) 261-271.

[25] Czajko J., Chaos, Solit. Fract. 21 (2004) 501-512.

[26] Geroch R., General relativity from A to B. Chicago: The Univ. of Chicago Press, 1978, pp. 166, 171. 
[27] O'Neill B., Semi-Riemannian geometry with applications to relativity. New York: Academic Press, 1983, p. 171.

[28] Czajko J., Chaos, Solit. Fract. 11 (2000) 1983-1992. 